\title{
Enhanced antitumor activity of cerulenin combined with oxaliplatin in human colon cancer cells
}

\author{
RISA SHIRAGAMI, SOICHIRO MURATA, CHIHIRO KOSUGI, TOHRU TEZUKA, MASATO YAMAZAKI, \\ ATSUSHI HIRANO, YUKINO YOSHIMURA, MASATO SUZUKI, KIYOHIKO SHUTO and KEIJI KODA
}

Department of Surgery, Teikyo University Chiba Medical Center, Ichihara, Chiba 299-0111, Japan

Received February 20, 2013; Accepted April 5, 2013

DOI: 10.3892/ijo.2013.1978

\begin{abstract}
Fatty acid synthase is highly expressed in many types of human cancers. Cerulenin, a natural inhibitor of fatty acid synthase, induced apoptosis in the human colon cancer cell lines HCT116 and RKO. Oxaliplatin also induced cell death in these cell lines. Cerulenin treatment was associated with reduced levels of phosphorylated Akt, activation of p38 and induced caspase- 3 cleavage and finally caused apoptosis. Oxaliplatin induced activation of the p53-p21 pathway and p38. In combination with cerulenin and oxaliplatin, activation of the p53-p21 pathway and p38 occurred in a smaller concentration and finally induced caspase-3 cleavage in a smaller concentration of cerulenin and oxaliplatin. In xenotransplanted SCID mice, the cerulenin + oxaliplatin group significantly inhibited tumor progression compared to the control, cerulenin and oxaliplatin groups. Based on these studies, inhibiting fatty acid synthase would be an effective strategy to treat unresectable colorectal cancer tumors in combination with oxaliplatin. Fatty acid synthase inhibitor would be one of the best counterparts of oxaliplatin, which reduces the dose and side-effects of oxaliplatin and would make it possible to endure the chemotherapy over a longer period.
\end{abstract}

\section{Introduction}

Colorectal cancer (CRC) is one of the most common cancers in the world and $\sim 90 \%$ of CRC deaths are caused by metastasis, not by primary solid tumors (1). Despite recent advances, systemic chemotherapy for metastatic disease is considered palliative and long-term survivors are rarely seen treated only by chemotherapy (2).

Overexpression of fatty acid synthase (FASN) is common in many human cancers and blocking FASN inhibits growth and leads to apoptosis in these cancer cells (3). Cerulenin is a small molecular FASN inhibitor, which has been isolated

Correspondence to: Dr Soichiro Murata, Department of Surgery, Teikyo University Chiba Medical Center, 3426-3 Anesaki, Ichihara, Chiba 299-0111, Japan

E-mail: soichiromurata@live.jp

Key words: cerulenin, oxaliplatin, colorectal cancer from Cephalosporium caerulens. Cerulenin contains an epoxy group that reacts with the ketoacyl synthase domain. It has revealed significant antitumor activity in various cancer cells by inducing apoptosis and growth inhibition (4).

Oxaliplatin is a platinum-based drug considered to be the most promising chemotherapy for CRC (5). Oxaliplatin treatment produces high levels of single- and double-strand breaks in DNA due to replication fork collapse and nuclease attack at the site of platinated cross-links and finally causes cell cycle arrest (6). Several clinical trials have shown, however, that oxaliplatin by itself is less effective compared to oxaliplatin combination therapy (7).

Our previous study showed the ability of cerulenin to cause cytotoxicity and induce apoptosis in murine CRC cells and in a murine xenograft model (8). In this study we reveal that cerulenin causes cytotoxicity of human CRC cell line HCT116 in vitro and in vivo. Next, we hypothesized that cerulenin can potentiate the cytotoxicity of oxaliplatin. In this study we report cerulenin and oxaliplatin have synergistic cytotoxicity and cerulenin can reduce the dosage of oxaliplatin in the treatment of human CRC.

\section{Materials and methods}

Reagents. Cerulenin and oxaliplatin were obtained from Sigma (St. Louis, MO, USA). For cell culture and i.p. injections, cerulenin was dissolved in acetone at a concentration of $20 \mathrm{mg} / \mathrm{ml}$ and stored at $-20^{\circ} \mathrm{C}$. Oxaliplatin was dissolved in sterile water. In in vitro experiments, $12.5-100 \mu \mathrm{M}$ of cerulenin and 0.5-2.5 $\mu \mathrm{M}$ of oxaliplatin were added to the medium. Cell viability assay and western blot experiments were performed $24 \mathrm{~h}$ later after adding cerulenin and oxaliplatin. In in vivo experiments, treatment with cerulenin at 15 and $30 \mathrm{mg} / \mathrm{kg}$ were given i.p. at days 7, 10, 14 and 17 after tumor inoculation. In in vivo treatment with oxaliplatin, 2.5 and $5 \mathrm{mg} / \mathrm{kg}$ of oxaliplatin were given i.p. at the same schedule as cerulenin.

Cell culture. The human CRC cell lines HCT116 and RKO were used and tested for mycoplasm-free cell lines. These cancer cells were subdivided in multiple tubes for stock in liquid nitrogen immediately after possession. All cell lines were subjected to the present experiment within 6 months of resuscitation. Stock cultures were grown in high-glucose DMEM containing $10 \%$ FBS and $1 \%$ antibiotics. The cells were grown 

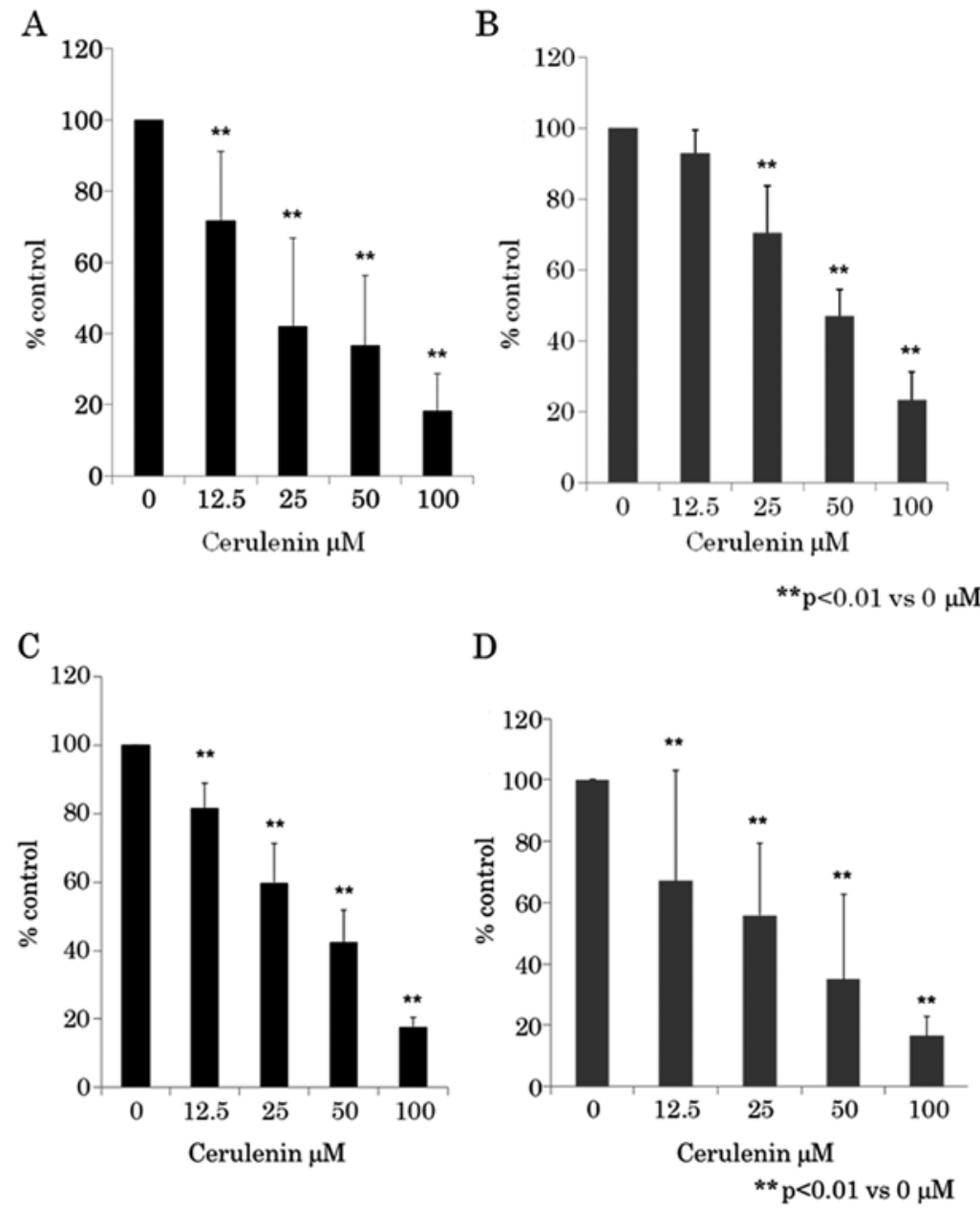

Figure 1. Effect of cerulenin on the WST-8 assay and BrdU assay of human CRC cell lines. CRC cell lines were treated with $0-100 \mu \mathrm{M}$ cerulenin for $24 \mathrm{~h}$. (A) WST-8 of HCT116; (B) WST-8 of RKO; (C) BrdU of HCT116; (D) BrdU of RKO. ${ }^{* *}$ p $<0.01$ compared to $0 \mu \mathrm{M}$ cerulenin. The values indicate ratio compared to $0 \mu \mathrm{M}$ cerulenin as $100 \%$.

in growth medium at $37^{\circ} \mathrm{C}$ in a $95 \%$ air, $5 \% \mathrm{CO}_{2}$-humidified incubator.

Cell viability assay. To measure the cytotoxicity of cerulenin against HCT116 and RKO cells, $3 \times 10^{3}$ cells were plated per well onto 96-well plates. Following overnight culture, cerulenin and oxaliplatin were added at specified concentrations. After $24 \mathrm{~h}$ of incubation, cell viability was measured by the mitochondrial activity in reducing 2-(2-methoxy-4nitrophenyl)-3-(4-nitrophenyl)-5-(2,4-disulfophenyl)-2H-tetrazolium monosodium salt (WST-8) to formazan using a Cell Counting kit-8 (Dojindo Laboratories, Kumamoto, Japan). Cells were incubated with a reagent according to the manufacturer's instructions. Plates were read at A450 on a spectrometer.

Cell proliferation assay. To measure the cell proliferation activity of cerulenin and oxaliplatin against HCT116 and RKO cancer cells, $3 \times 10^{3}$ cells were plated per well onto 96-well plates. Following overnight culture, cerulenin and oxaliplatin were added at specified concentrations. After $24 \mathrm{~h}$ of incubation, cell proliferation was measured with a BrdU assay kit (Roche Diagnostics, Penzberg, Germany). Cells were incubated with a reagent as per the manufacturer's instructions. Plates were read at A450 on a spectrometer.
Apoptosis assay. The In situ Cell Death Detection kit (Roche Diagnostics, Basel, Switzerland) was used for the demonstration of apoptotic cell death of cell culture. Cells $\left(3 \times 10^{4}\right)$ were plated per well onto Lab-Tek II Chamber Slides (Nalge Nunc International, Tokyo, Japan) and were incubated with the terminal deoxynucleotidyl transferase-mediated dUTP nick-end labeling (TUNEL) reaction mixture according to the manufacturer's recommendations.

Western blot analysis. For western blot analysis, total protein extracts of HCT116 cells were obtained $24 \mathrm{~h}$ after cerulenin and oxaliplatin treatment and separated by $10 \%$ SDS-PAGE and transferred to nitrocellulose membrane (Millipore, Bedford, MA, USA). The following antibodies were used as primary antibodies: total Akt (9272), phosphoserine 473 Akt (9271), cleaved caspase-3 (9661), phospho-p38 (4511p), phosphoserine 15 p53 (9284p), p21waf1 (2947p) and glyceraldehyde-3-phosphate dehydrogenase (GAPDH) (2118) (Cell Signaling Technology, Beverly, MA, USA). Purified mouse antiFAS antibody (610962) was purchased from BD Biosciences (San Jose, CA, USA). Secondary goat anti-rabbit and goat anti-mouse antibodies conjugated with horseradish peroxidase were purchased from Cell Signaling Technology. Immunoblots were analyzed by enhanced chemiluminescence. 

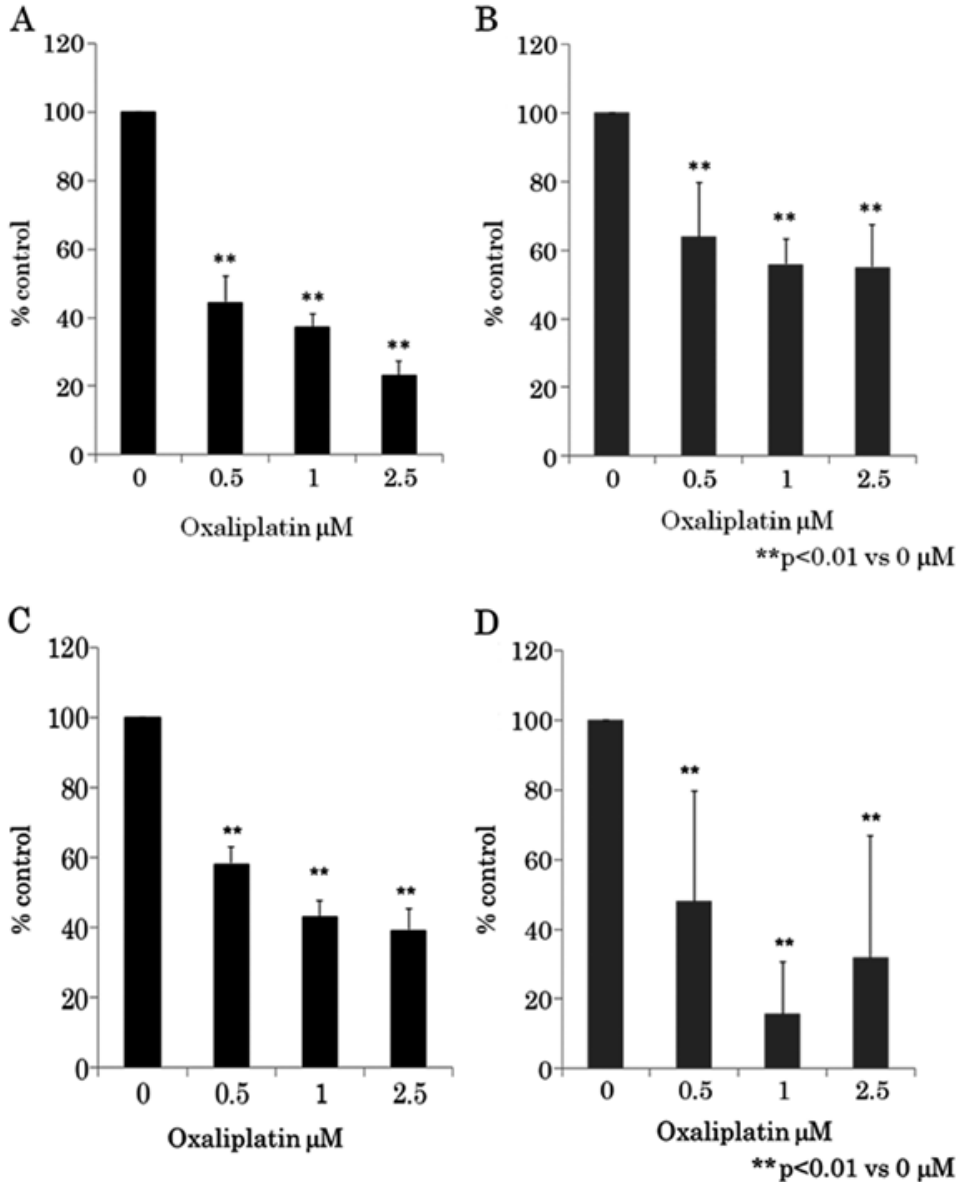

Figure 2. Effect of oxaliplatin on the WST-8 assay and BrdU assay of human CRC cell lines. CRC cell lines were treated with 0-2.5 $\mu \mathrm{M}$ cerulenin for $24 \mathrm{~h}$ (A) WST-8 of HCT116; (B) WST-8 of RKO; (C) BrdU of HCT116; (D) BrdU of RKO. ${ }^{* *}$ p $<0.01$ compared to $0 \mu \mathrm{M}$ oxaliplatin. The values indicate ratio compared to $0 \mu \mathrm{M}$ oxaliplatin as $100 \%$.

Animals. Eight-week-old male severe combined immunodeficiency (SCID) mice (Clea, Tokyo, Japan), weighing 24-28 g were utilized. The mice were kept in a temperature-controlled room on a 12-h light-dark cycle. They had free access to water and standard chow throughout the experiment. After an acclimation period of $\geq 7$ days, the mice were separated into four groups as follows: control group, mice without any treatment $(n=12)$; cerulenin group, mice with cerulenin treatment $15(\mathrm{n}=5)$ and $30 \mathrm{mg} / \mathrm{kg}(\mathrm{n}=5)$; oxaliplatin group, mice with oxaliplatin treatment $2.5 \quad(\mathrm{n}=8)$ and $5 \mathrm{mg} / \mathrm{kg}(\mathrm{n}=5)$; and combination group, mice with $15 \mathrm{mg} / \mathrm{kg}$ of cerulenin and $2.5 \mathrm{mg} / \mathrm{kg}$ of oxaliplatin treatment $(\mathrm{n}=10)$. All animal experiments were carried out in a humane manner after receiving approval from the Institutional Animal Committee of Teikyo University and in accordance with the Regulation for Animal Experiments of the University and Fundamental Guidelines for Proper Conduct of Animal Experiments and Related Activities in Academic Research Institutions under the jurisdiction of the Ministry of Education, Culture, Sports, Science and Technology of Japan.

Xenograft. Cells $\left(2 \times 10^{6}\right)$ of HCT116 were injected subcutaneously into the right flank of each mouse with a 27-gauge needle. Tumors were detected by palpation and measured periodically with calipers. Seven days after tumor injection, cerulenin and oxaliplatin were injected intraperitoneally every 3 days. Twenty-one days after inoculation, the mice were sacrificed and tumors were removed for examination. Tumor tissue, fixed in $10 \%$ buffered formalin, was used for histological analyses.

Statistical analysis. All data are expressed as the mean \pm SD of samples. Comparisons between various points were made using one-way ANOVA. Significant data were examined by the Bonferroni-Dunn multiple comparisons post hoc test. In all cases, $\mathrm{P}<0.05$ was considered significant.

\section{Results}

Dose-dependent inhibition of proliferation of human CRC cell lines by cerulenin. We initially determined whether cerulenin treatment led to the inhibition of human CRC cell proliferation. CRC cells were treated with various doses of cerulenin for $24 \mathrm{~h}$ and cell viability was assayed using WST-8 assay (Fig. 1A and B) and BrdU assay (Fig. 1C and D). Fig. 1 shows that as the dose of cerulenin increased from 12.5 to $100 \mu \mathrm{M}$, cell growth inhibition increased in a dose-dependent manner in CRC cell lines HCT116 and RKO. Cerulenin-induced growth inhibition was found to be statistically significant $(\mathrm{p}<0.01)$ (one-way ANOVA) in $12.5-100 \mu \mathrm{M}$ of cerulenin compared to $0 \mu \mathrm{M}$. 


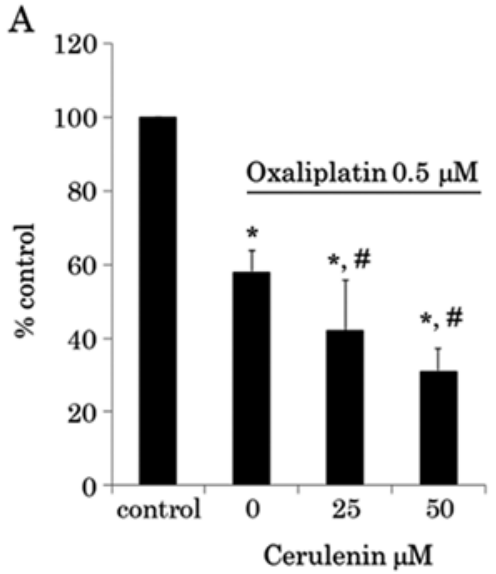

C

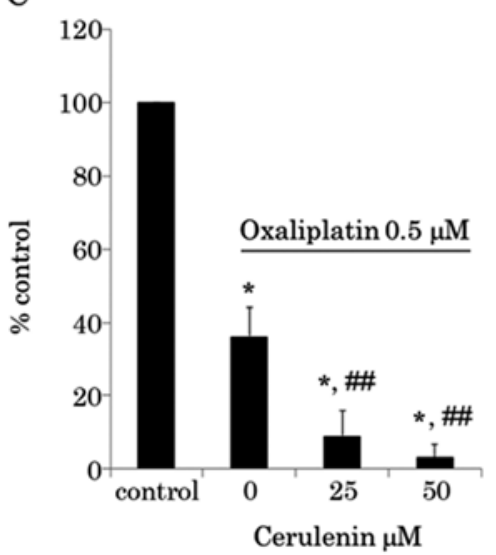

B

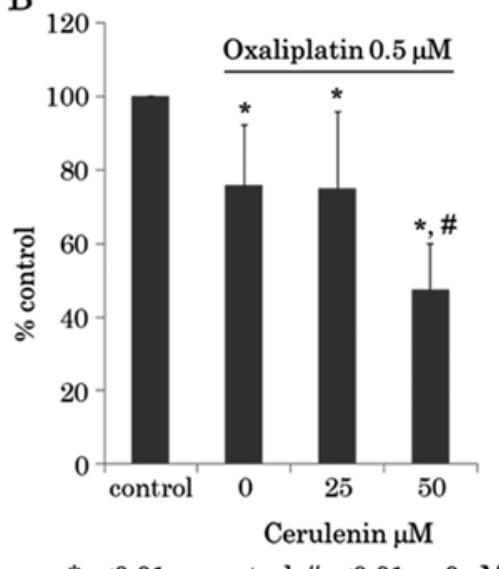

${ }^{*} \mathrm{p}<0.01$ vs control, $\# \mathrm{p}<0.01$ vs $0 \mu \mathrm{M}$

$\mathrm{D}$

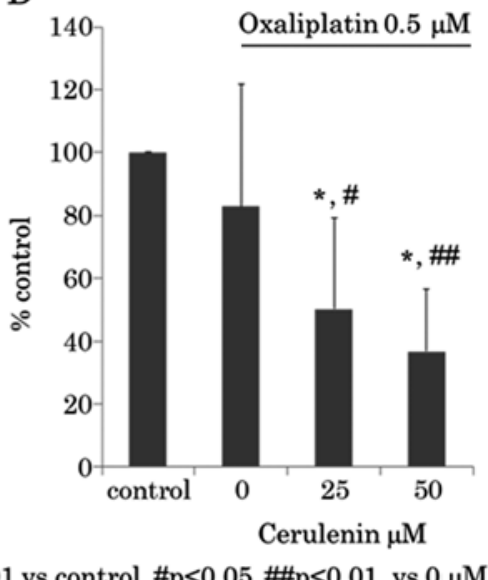

Figure 3. Synergistic effect of oxaliplatin added to cerulenin in WST-8 assay and BrdU assay of human CRC cell lines. CRC cell lines were treated with $0.5 \mu \mathrm{M}$ oxaliplatin, then $0-50 \mu \mathrm{M}$ cerulenin was added. Twenty-four hours later, WST- 8 and BrdU assays were performed. (A) WST-8 of HCT116; (B) WST-8 of RKO; (C) BrdU of HCT116; (D) BrdU of RKO. Control group, without any treatment. " $\mathrm{p}<0.01$ compared to control. ${ }^{\#} \mathrm{p}<0.05$ and ${ }^{\# \#} \mathrm{p}<0.01$ compared to $0 \mu \mathrm{M}$ cerulenin. The values indicate ratio compared to control as $100 \%$.

Dose-dependent inhibition of proliferation of human CRC cell lines by oxaliplatin. Next, we determined whether oxaliplatin treatment led to the inhibition of human CRC cell proliferation. CRC cells were treated with various doses of oxaliplatin for $24 \mathrm{~h}$ and cell viability was assayed using WST-8 assay (Fig. 2A and B) and BrdU assay (Fig. 2Cand D). Fig. 2 shows that as the dose of oxaliplatin increased from 0.5 to $2.5 \mu \mathrm{M}$, cell growth inhibition increased in a dose-dependent manner in CRC cell lines HCT116 and RKO. Oxaliplatin-induced growth inhibition was found to be statistically significant (p<0.01) (one-way ANOVA) in 0.5-2.5 $\mu \mathrm{M}$ of oxaliplatin compared to $0 \mu \mathrm{M}$.

Synergistic antitumor effect between cerulenin and oxaliplatin. Next we determined whether a synergistic antitumor effect exists between cerulenin and oxaliplatin. The cerulenin effect under $0.5 \mu \mathrm{M}$ of oxaliplatin was evaluated using WST-8 assay (Fig. 3A and B) and BrdU assay (Fig. 3C and D). The oxaliplatin effect under $25 \mu \mathrm{M}$ of cerulenin was evaluated using WST-8 assay (Fig. 4A and B) and BrdU assay (Fig. 4C and D). The results indicated that cerulenin and oxaliplatin have synergic antitumor effects.
Induction of apoptosis via activation of caspase-dependent pathway by cerulenin combined with oxaliplatin. In subsequent experiments, we determined the mechanism of the observed suppressive effect of combination therapy by WST-8 and BrdU assays. The overexpression of FASN has been observed to cooperate with survival pathways, including the phosphatidylinositol-3-kinase (PI3K)/Akt pathway. HCT116 cells expressed FASN and p-Akt constitutively and treatment of cerulenin suppressed FAS expression in $100 \mu \mathrm{M}$ of cerulenin as previously reported by us (data not shown). Dephosphorylated constitutive activated Akt, activation of p38 and increased cleaved caspase- 3 in cerulenin treatment (Fig. 5A). Oxaliplatin induced p53-p21 pathway and p38, but did not increase cleaved caspase-3 (Fig. 5B). In combination therapy, p53-p21 pathway and p38 activation occurred in a lower dose and induced caspase-3 cleavage (Fig. 5C).

Apoptotic effect of HCT116 by cerulenin and oxaliplatin combination therapy. TUNEL staining of HCT116 cells shows apoptotic cells in $25 \mu \mathrm{M}$ of cerulenin and $0.5 \mu \mathrm{M}$ of oxaliplatin. Combination with cerulenin and oxaliplatin induced apoptosis significantly (Fig. 6). 
A

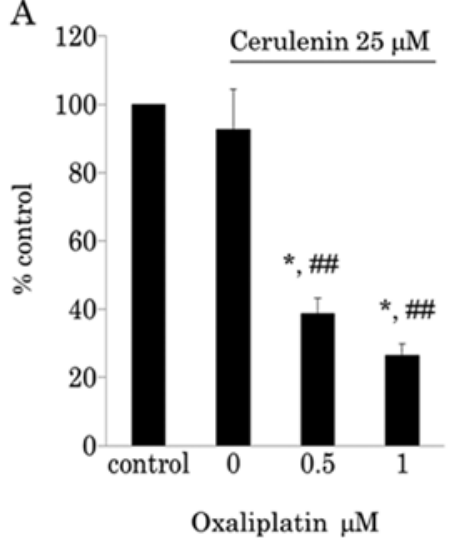

B

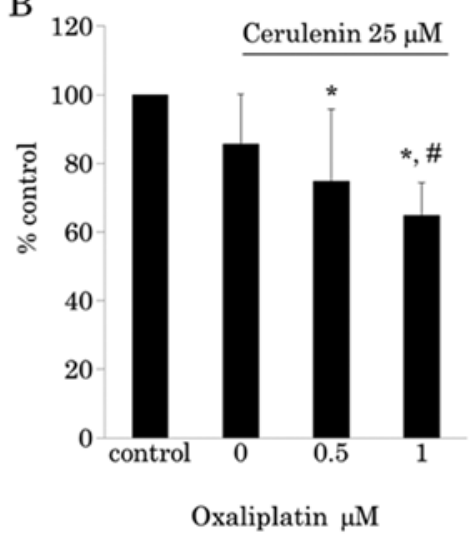

${ }^{*} \mathrm{p}<0.01$ vs control, \#p<0.05,\#p<0.01 vs $0 \mu \mathrm{M}$
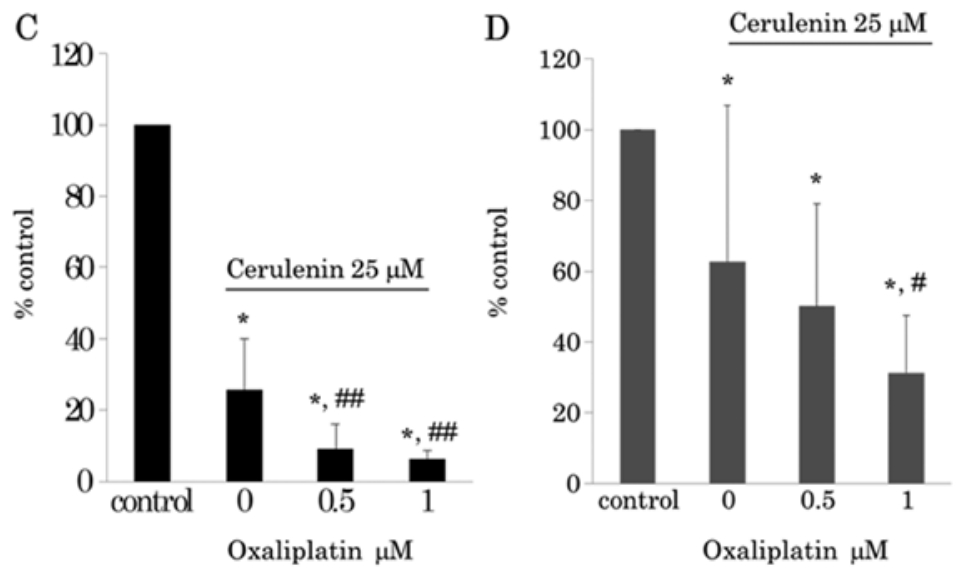

${ }^{*} \mathrm{p}<0.01$ vs control, \#p<0.05,\#p<0.01 vs $0 \mu \mathrm{M}$

Figure 4. Synergistic effect of cerulenin added to oxaliplatin in WST-8 assay and BrdU assay of human CRC cell lines. CRC cell lines were treated with $25 \mu \mathrm{M}$ cerulenin, then $0-1 \mu \mathrm{M}$ oxaliplatin was added. Twenty-four hours later, WST-8 and BrdU assays were performed. (A) WST-8 of HCT116; (B) WST-8 of RKO; (C) BrdU of HCT116; (D) BrdU of RKO. Control group, without any treatment. ${ }^{*} \mathrm{p}<0.01$ compared to control. ${ }^{\#} \mathrm{p}<0.05$ and ${ }^{\# \#} \mathrm{p}<0.01$ compared to $0 \mu \mathrm{M}$ oxaliplatin. The values indicate ratio compared to control as $100 \%$.
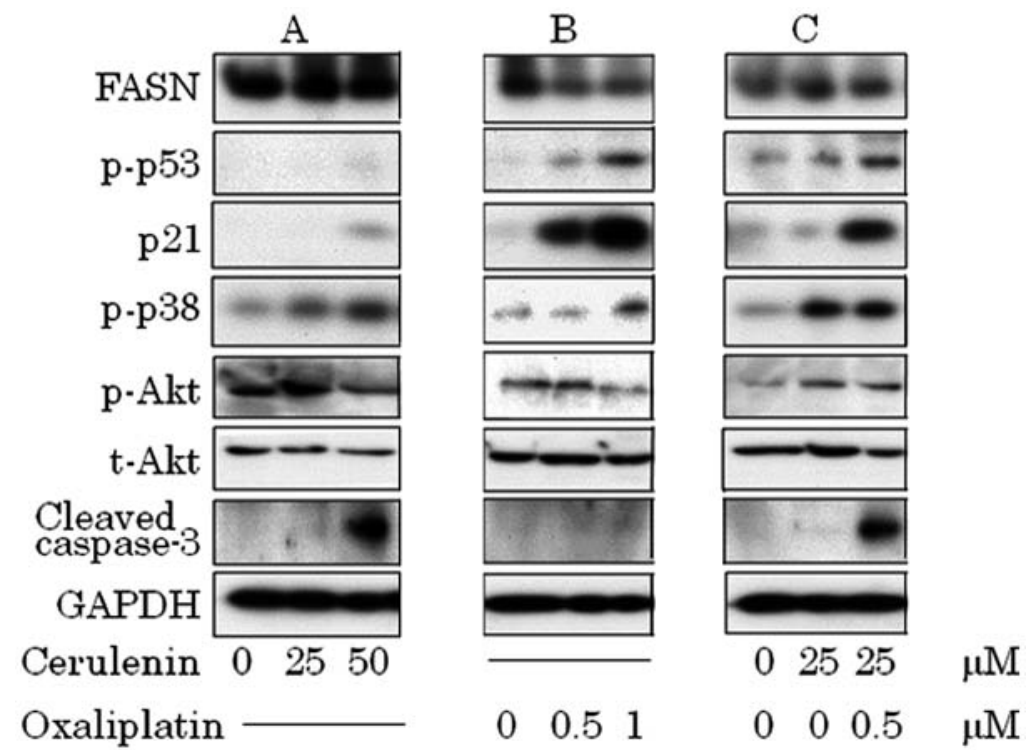

Figure 5. (A) Cerulenin treatment causes $\mathrm{p} 38$ phosphorylation, dephosphorylation of constitutive phosphorylation of Akt and accumulation of cleaved caspase-3. (B) Oxaliplatin treatment causes p53 phosphorylation, and p21 activation. (C) In combination, activation of p53-p21 pathway, p38 and caspase-3 cleavage. (A) HCT116 was treated with 0,25 and $50 \mu \mathrm{M}$ of cerulenin for $24 \mathrm{~h}$. (B) HCT116 was treated with $0,0.5$ and $1.0 \mu \mathrm{M}$ of oxaliplatin for $24 \mathrm{~h}$. (C) HCT116 was treated with $25 \mu \mathrm{M}$ of cerulenin combined with $0.5 \mu \mathrm{M}$ of oxaliplatin. After cell lysis, equal amounts of proteins were separated by SDS-PAGE, transferred to Immobilon membrane and immunoblotted with antibodies against FASN, p-p53, p21, p-p38, p-Akt, t-Akt, cleaved caspase-3 and GAPDH as indicated. 


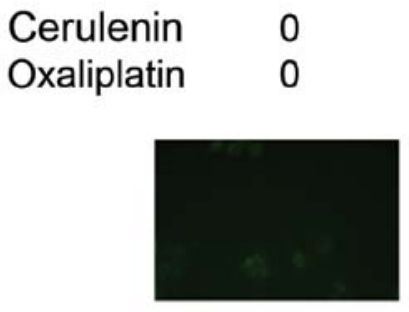

25

0

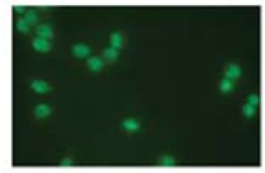

0

0.5

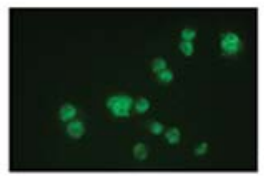

25

$0.5 \mu \mathrm{M}$

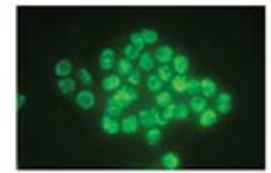

Figure 6. Cerulenin and oxaliplatin combination causes apoptosis in HCT116. TUNEL staining of HCT116 cells treated with $25 \mu \mathrm{M}$ cerulenin, $0.5 \mu \mathrm{M}$ oxaliplatin and combined with $25 \mu \mathrm{M}$ cerulenin and $0.5 \mu \mathrm{M}$ oxaliplatin for $24 \mathrm{~h}$, are shown. Stained cells indicate TUNEL-positive apoptotic cells.

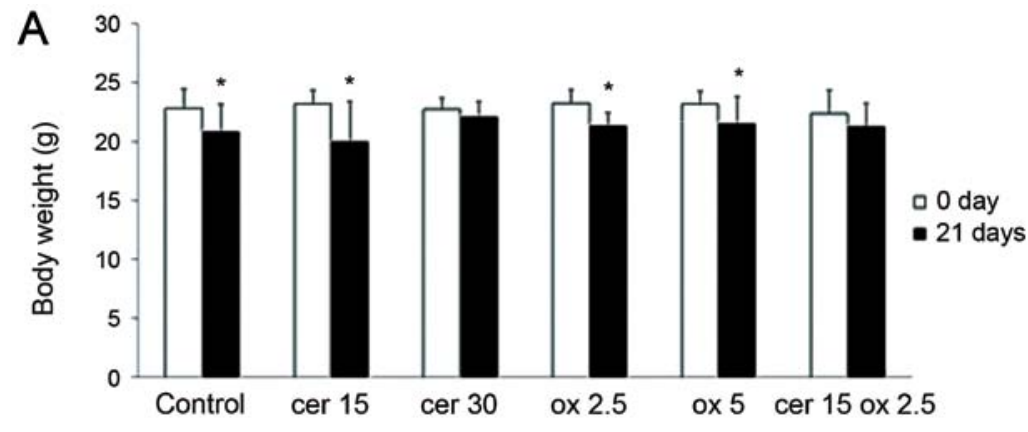

B
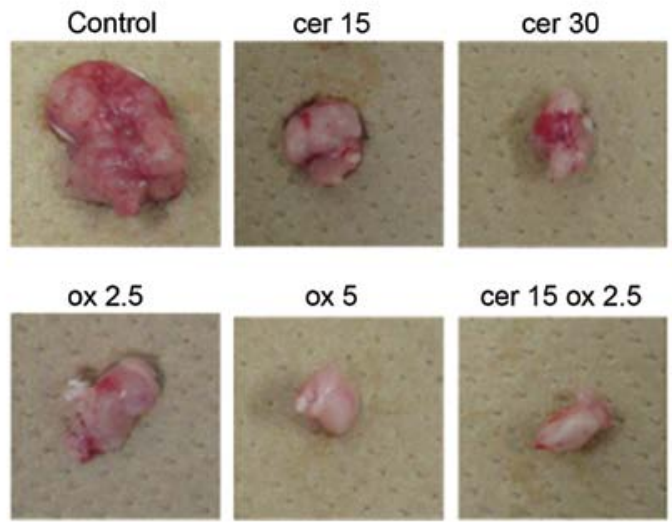

ox 5

cer 15 ox 2.5
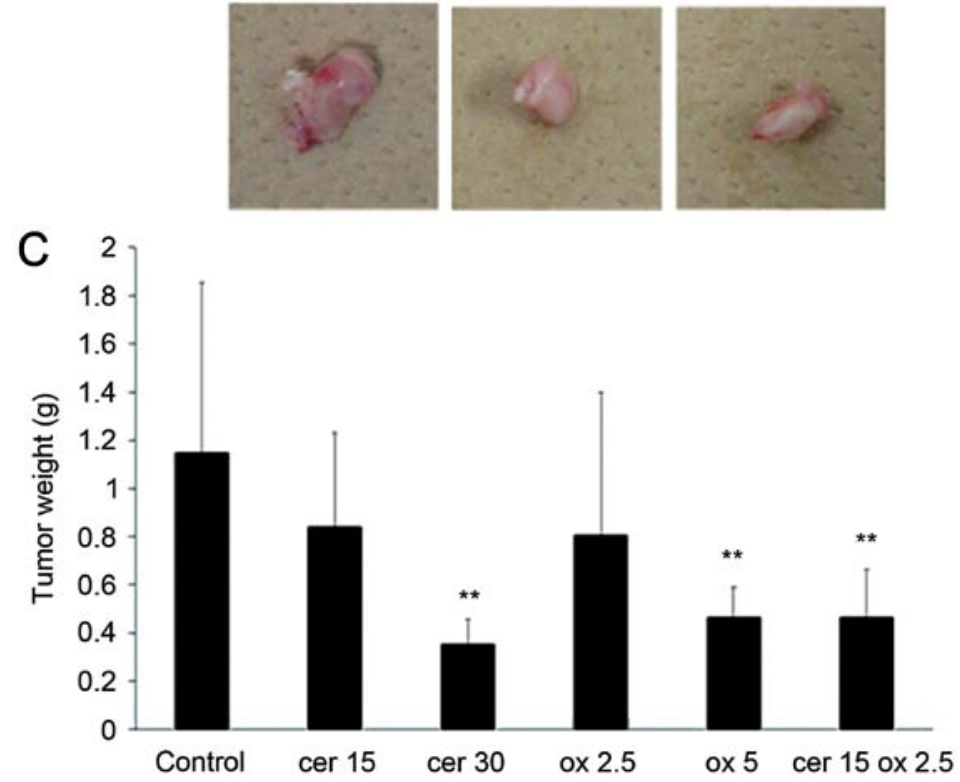

Figure 7. Cerulenin and oxaliplatin combination therapy significantly inhibits tumor growth of the xenograft HCT116 tumors in SCID mice. HCT116 was injected to the right flank subcutaneously. Seven days later, cerulenin 15 and $30 \mathrm{mg} / \mathrm{kg}$, oxaliplatin 2.5 and $5 \mathrm{mg} / \mathrm{kg}$ and cerulenin $15 \mathrm{mg} / \mathrm{kg}$ and oxaliplatin $2.5 \mathrm{mg} / \mathrm{kg}$ were treated 4 times. cer 15 , cerulenin $15 \mathrm{mg} / \mathrm{kg}$; cer 30 , cerulenin $30 \mathrm{mg} / \mathrm{kg}$; ox 2.5, oxaliplatin $2.5 \mathrm{mg} / \mathrm{kg}$; ox 5 , oxaliplatin $5 \mathrm{mg} / \mathrm{kg}$; cer 15 ox 2.5 , cerulenin $15 \mathrm{mg} / \mathrm{kg}$ and oxaliplatin $2.5 \mathrm{mg} / \mathrm{kg}$. (A) Weight of animals in 6 groups. Columns, mean; bars, SD. White bar, on the day of tumor injection. Black bar, 21 days after tumor inoculation. "p $<0.05$ versus 0 day of control group, t-test. (B) Representative xenograft tumors of the 6 groups. (C) Tumor weight of the 6 groups. ${ }^{* *} \mathrm{p}<0.01$ versus control group.

Cerulenin combined with oxaliplatin inhibits tumor growth of HCT116 xenografts. We evaluated the potential effectiveness of cerulenin and oxaliplatin combination for a xenograft model of HCT116, subcutaneously injected into the right flank of each mouse. Fig. 7A shows the weight of animals in control, cerulenin $15 \mathrm{mg} / \mathrm{kg}$ (cer 15) and $30 \mathrm{mg} / \mathrm{kg}$ (cer 30), oxaliplatin 


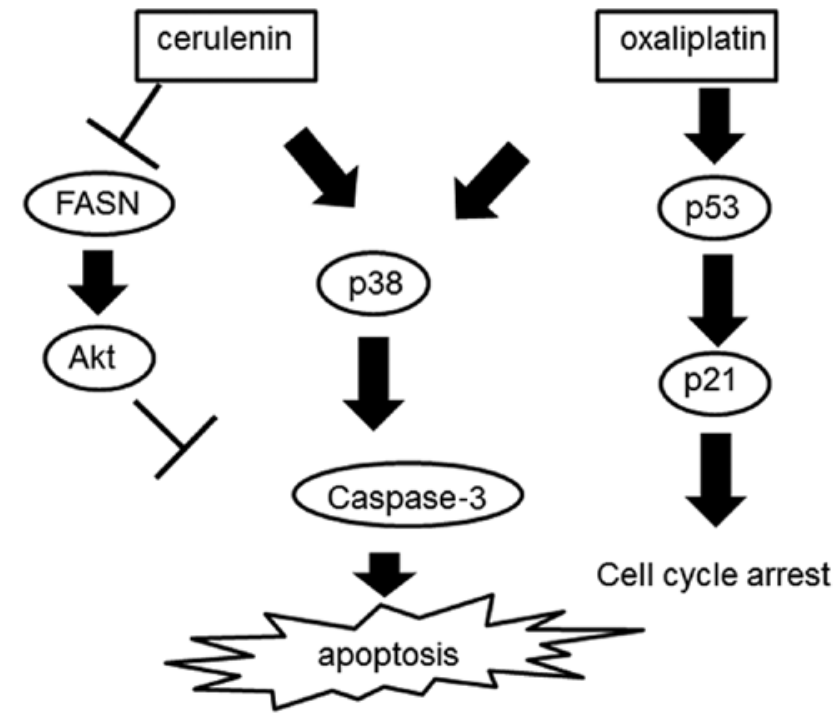

Figure 8 . The scheme of the combination therapy consists of cerulenin and oxaliplatin. Cerulenin inhibits FASN, and Akt activates p38 to induce apoptosis. Oxaliplatin induces the p53-p21 pathway causing cell cycle arrest without activating the apoptotic pathways. In combination with cerulenin and oxaliplatin, p53-p21 pathway and p38 were activated, which causes cell cycle arrest and apoptosis.

$2.5 \mathrm{mg} / \mathrm{kg}$ (ox 2.5) and $5 \mathrm{mg} / \mathrm{kg}$ (ox 5) and combination group, with $15 \mathrm{mg} / \mathrm{kg}$ of cerulenin and $2.5 \mathrm{mg} / \mathrm{kg}$ of oxaliplatin (cer 15 ox 2.5). In control, cer 15, ox 2.5 and ox 5 groups, significant body weight loss was observed during treatment. However, in cer 15 ox 2.5 group, significant weight loss was not observed. Fig. 7B shows tumors removed from the representative control, cer 15 , cer 30 , ox 2.5 , ox 5 and cer 15 ox 2.5 groups. Tumor growth was significantly inhibited in the cer 30 and ox 5 group compared to the control group. In cer 15 ox 2.5 group, the tumor growth was inhibited at the same level compared to cer 30 and ox 5. Fig. 7C indicates tumor weight in the 6 groups. Tumor growth was significantly reduced in cer 30 , ox 5 and cer 15 ox 2.5 groups compared to control group.

\section{Discussion}

The role of increased FASN in cancer cells and the mechanisms of cell killing by inhibitors of FASN are still not fully understood (9). The effect of an intermediate metabolite of fatty acid synthesis on cancer cells is likely mediated through cell signaling pathways, especially Akt (10). The overexpression of FASN has been observed to cooperate with survival pathways including the phosphatidylinositol-3-kinase (PI3K)/Akt pathway. HCT116 expressed FASN and p-Akt constitutively and treatment with cerulenin suppressed FASN expression, dephosphorylated constitutive activated Akt, activated p38 and increased cleaved caspase-3, finally causing apoptosis.

p38 MAP kinase is a member of the MAP kinase family and is activated by a variety of cellular stresses including osmotic shock, inflammatory cytokines, lipopolysaccharide, UV light and growth factors (11-15). Activated p38 MAP kinase appears to have multiple targets in the apoptotic pathway. In nitric oxide (NO)-induced neuronal apoptosis, p38 MAP kinase activates caspase and induces apoptosis (16). C75 is one of the FASN inhibitors reported to induce p38 activation (17). Oxaliplatin activates p38 MAP kinase phosphorylation in human colon carcinoma cells (18).

The p53 protein, a well-characterized tumor suppressor, plays a pivotal role in the maintenance of genomic stability $(19,20)$. Activation of p53 can lead to either cell cycle arrest and DNA repair or apoptosis (21). DNA damage induces phosphorylation of p53 at Ser15 and phosphorylation promotes both the accumulation and activation of p53 in response to DNA damage (22). Activated p53 up-regulates p21 transcription (23). HCT116 cell line, which harbors a wild-type p53 protein, is sensitive to oxaliplatin treatment (24). The p53-p21 pathway is a major determinant of sensitivity to oxaliplatin of the p53 wild-type HCT116 cell line (25). The p53-p21 pathway leads to cell cycle arrest without apoptosis (26). Based on our results, oxaliplatin induces the p53-p21 pathway, causing cell cycle arrest without activating apoptotic pathways. In combination with cerulenin and oxaliplatin, p53-p21 pathway and p38 were activated, which causes cell cycle arrest and apoptosis. In a xenotransplant mouse model, the combination therapy, which consists of $2.5 \mathrm{mg} / \mathrm{kg}$ of oxaliplatin and $15 \mathrm{mg} / \mathrm{kg}$ of cerulenin, had the same tumor shrinkage effect compared to the oxaliplatin $5 \mathrm{mg} / \mathrm{kg}$ group, which means that by adding cerulenin oxaliplatin dose could be reduced. In the combination therapy, mitotic figures of the tumor were significantly decreased and TUNEL-positive cancer cells were increased. Fig. 8 shows the scheme of the combination therapy consisting of cerulenin and oxaliplatin.

Oxaliplatin is a most promising chemotherapeutic agent, which consists of FOLFOX for unresectable CRC (5). Neurotoxicity is a severe and treatment-limiting side-effect of several chemotherapeutic agents (27). Sensory neurotoxicity is a potentially limiting factor in many patients who might otherwise achieve good results with oxaliplatin therapy (28). In patients with severe neurotoxicity, reduction or discontinuation of oxaliplatin is often required. This study revealed that cerulenin can potentiate oxaliplatin in in vitro and in vivo. Cerulenin is one of the better combinations with oxaliplatin, which achieves reduction of oxaliplatin and long-term tolerated chemotherapy for unresectable CRC.

In conclusion, cerulenin has a cytotoxic effect on human CRC cell line HCT116. Moreover, cerulenin potentiated cytotoxicity of oxaliplatin. Cerulenin would be effective in treatment of unresectable CRC in combination with oxaliplatin, which reduces the dose of oxaliplatin and would make it possible to endure the chemotherapy over a longer period.

\section{Acknowledgements}

The authors thank Satoko Nakabayashi for technical assistance. This study was supported in part by grants-in-aid from the Ministry of Education, Culture, Sports, Science and Technology of Japan (MEXT).

\section{References}

1. Gupta GP and Massague J: Cancer metastasis: building a framework. Cell 127: 679-695, 2006.

2. Tachimori A, Yamada N, Amano R, et al: Combination therapy of S-1 with selective cyclooxygenase-2 inhibitor for liver metastasis of colorectal carcinoma. Anticancer Res 28: 629-638, 2008.

3. Kuhajda FP: Fatty acid synthase and cancer: new application of an old pathway. Cancer Res 66: 5977-5980, 2006. 
4. Elbaz A, Wu X, Rivas D, et al: Inhibition of fatty acid biosynthesis prevents adipocyte lipotoxicity on human osteoblasts in vitro. J Cell Mol Med 14: 982-991, 2010.

5. Wils J: Adjuvant treatment of colon cancer: past, present and future. J Cemother 19: 115-122, 2007.

6. Pires IM, Ward TH and Dive C: Oxaliplatin responses in colorectal cancer cells are modulated by CHK2 kinase inhibitors. Br J Pharmacol 159: 1326-1338, 2010.

7. Ramanathan RK, Clark JW, Kemeny NE, et al: Safety and toxicity analysis of oxaliplatin combined with fluorouracil or as a single agent in patients with previously treated advanced colorectal cancer. J Clin Oncol 21: 2904-2911, 2003.

8. Murata S, Yanagisawa K, Fukunaga K, Oda T, Kobayashi A, Sasaki R and Ohkohchi N: Fatty acid synthase inhibitor cerulenin suppresses liver metastasis of colon cancer in mice. Cancer Sci 101: 1861-1865, 2010.

9. Orita H, Coulter J, Lemmon C, et al: Selective inhibition of fatty acid synthase for lung cancer treatment. Clin Cancer Res 13 7139-7145, 2007

10. Wang HQ, Altomare DA, Skele KL, et al: Positive feedback regulation between AKT activation and fatty acid synthase expression in ovarian carcinoma cells. Oncogene 24: 3574-3582, 2005.

11. Rouse J, Cohen P, Trigon S, et al: A novel kinase cascade triggered by stress and heat shock that stimulates MAPKAP kinase-2 and phosphorylation of the small heat shock proteins. Cell 78: 1027-1037, 1994

12. Han J, Lee JD, Bibbs L and Ulevitch RJ: A MAP kinase targeted by endotoxin and hyperosmolarity in mammalian cells. Science 265: 808-811, 1994

13. Lee JC, Laydon JT, McDonnell PC, et al: A protein kinase involved in the regulation of inflammatory cytokine biosynthesis. Nature 372: 739-746, 1994.

14. Freshney NW, Rawlinson L, Guesdon F, et al: Interleukin-1 activates a novel protein kinase cascade that results in the phosphorylation of Hsp27. Cell 78: 1039-1049, 1994.

15. Raingeaud J, Gupta S, Rogers JS, et al: Pro-inflammatory cytokines and environmental stress cause p38 mitogen-activated protein kinase activation by dual phosphorylation on tyrosine and threonine. J Biol Chem 270: 7420-7426, 1995.
16. Ghatan S, Larner S, Kinoshita Y, et al: p38 MAP kinase mediates Bax translocation in nitric oxide-induced apoptosis in neurons. J Cell Biol 150: 335-347, 2000.

17. Gao Y, Lin LP, Zhu CH, Chen Y, Hou YT and Ding J: Growth arrest induced by $\mathrm{C} 75$, A fatty acid synthase inhibitor, was partially modulated by p38 MAPK but not by p53 in human hepatocellular carcinoma. Cancer Biol Ther 8: 978-985, 2006.

18. Liu HF, Hu HC and Chao JI: Oxaliplatin down-regulates survivin by p38 MAP kinase and proteasome in human colon cancer cells. Chem Biol Interact 188: 535-545, 2010.

19. Schwartz D and Rotter V: p53-dependent cell cycle control: response to genotoxic stress. Semin Cancer Biol 8: 325-336, 1998.

20. Taylor WR and Stark GR: Regulation of the G2/M transition by p53. Oncogene 20: 1803-1815, 2001

21. Levine AJ: p53, the cellular gatekeeper for growth and division. Cell 88: 323-331, 1997.

22. Shieh SY, Ikeda M, Taya Y and Prives C: DNA damage-induced phosphorylation of p53 alleviates inhibition by MDM2. Cell 91: 325-334, 1997.

23. Wang Y and Prives C: Increased and altered DNA binding of human $\mathrm{p} 53$ by $\mathrm{S}$ and $\mathrm{G} 2 / \mathrm{M}$ but not G1 cyclin-dependent kinases. Nature 376: 88-91, 1995

24. Gourdier I, Del Rio M, Crabbe L, et al: Drug specific resistance to oxaliplatin is associated with apoptosis defect in a cellular model of colon carcinoma. FEBS Lett 529: 232-236, 2002.

25. Toscano F, Parmentier B, Fajoui ZE, et al: p53 dependent and independent sensitivity to oxaliplatin of colon cancer cells. Biochem Pharmacol 74: 392-406, 2007.

26. Schwartz GK: Development of cell cycle active drugs for the treatment of gastrointestinal cancers: a new approach to cancer therapy. J Clin Oncol 23: 4499-4508, 2005.

27. Benett BK, Park SB, Lin CSY, et al: Impact of oxaliplatininduced neuropathy: a patient perspective. Support Care Cancer 20: 2959-2967, 2012.

28. Gœbel FM, Tournigand C, André T, et al: Oxaliplatin reintroduction in patients previously treated with leucovorin, fluorouracil and oxaliplatin for metastatic colorectal cancer. Ann Oncol 15 1210-1214, 2004. 